\title{
Primary Cutaneous Cryptococcosis in an Older Immunocompetent Patient: A Case Report
}

\author{
Hoon Choi, Yong Il Kim, Chan Ho Na, Min Sung Kim, Bong Seok Shin \\ Department of Dermatology, Chosun University College of Medicine, Gwangju, Korea
}

Corresponding Author:

Bong Seok Shin, $\mathrm{MD}, \mathrm{PhD}$

Department of Dermatology, Chosun

University College of Medicine, 309

Pilmun-daero, Dong-gu, Gwangju

61452, Korea

Email: derm75@chosun.ac.kr

ORCID:

https://orcid.org/0000-0001-9618-1763

Received: March 18, 2020

Revised: May 2, 2020

Accepted: May 4, 2020
Cryptococcus neoformans is an encapsulated yeast that can be found in pigeon droppings, hay, and dust. Primary cutaneous cryptococcosis (PCC) without systemic involvement is recognized as a distinct clinical condition and is rarely reported in immunocompetent patients. A 78-year-old woman with no history of other diseases except for hypertension presented with a painful diffuse erythematous plaque along with oozing on left forearm that had lasted for 7 weeks. She was treated with cefoperazone/sulbactam for 3 weeks under suspicion of bacterial cellulitis, although the lesions aggravated without any improvement. We performed bacterial and fungal cultures as well as incisional biopsy. The pathogen was identified as Cryptococcus neoformans following sequence analysis of the internal transcribed spacer gene. The patient was treated with fluconazole $400 \mathrm{mg} /$ day for 3 months, and there was no evidence of recurrence after 3 months of follow-up.

Key Words: Cryptococcus neoformans infection, Fungal skin diseases

\section{INTRODUCTION}

Cryptococcus neoformans is an encapsulated yeast that can be found in decaying wood, hay, dust, and feces of birds, especially pigeons. ${ }^{1)}$ Cryptococcosis is considered an opportunistic infection in immunocompromised people, such as patients with malignancy, human immunodeficiency virus (HIV) infection, and those on long-term treatment with glucocorticoids and immunosuppressive agents after organ transplantation. ${ }^{2)}$ The infections mainly involve the lungs and central nervous system, with skin invasion considered a sign of dissemination. ${ }^{3)}$ Primary cutaneous cryptococcosis (PCC) without systemic involvement is recognized as a distinctive clinical disease and is rarely reported in immunocompetent patients. ${ }^{4)}$ Herein, we report a case of PCC misdiagnosed as cellulitis in an older immunocompetent patient.

\section{CASE REPORT}

A 78-year-old woman living in rural South Korea and with no history of other diseases except for hypertension presented with a painful erythematous plaque with oozing ulcers on the left forearm that had lasted for 7 weeks (Fig. 1). She did not complain of any systemic symptoms such as fever and myalgia. Before the lesions developed, she was injured by a sickle while cutting grass. She was treated for 3 weeks with cefoperazone/sulbactam under the suspicion of cellulitis; however, the lesions became wider and worsened. We performed bacterial and fungal cultures, as well as an incisional biopsy. The biopsy specimen was stained with the following: Gomori methenamine silver stain (GMS), periodic acid-Schiff stain (PAS), and acid-fast stain. Histopathologically, granulomatous inflammation with aggregated histiocytes and multinucleated giant cells were observed in the dermis, along with multiple intracytoplasmic yeasts (Fig. 2A, 2B). Furthermore, the yeasts were also identified on staining with GMS and PAS (Fig. 2C, 2D). No specific findings were observed on other investigations such as complete blood cell count; serum chemistry analysis; and tests for HIV infection, beta-D-glucan, and C. neoformans antigen in the blood. C-reactive protein level was elevated at $3.95 \mathrm{mg} / \mathrm{dL}$. No abnormalities were observed on radiography of the chest, computed tomography (CT) of the thorax and brain, and cerebrospinal fluid (CSF) 
examinations for evaluation of systemic cryptococcosis. The patient was treated with intravenous fluconazole at doses of $400 \mathrm{mg}$ / day for 4 days and $200 \mathrm{mg}$ /day for 10 days. Two weeks later, several cream-colored smooth colonies were observed in cultures, which

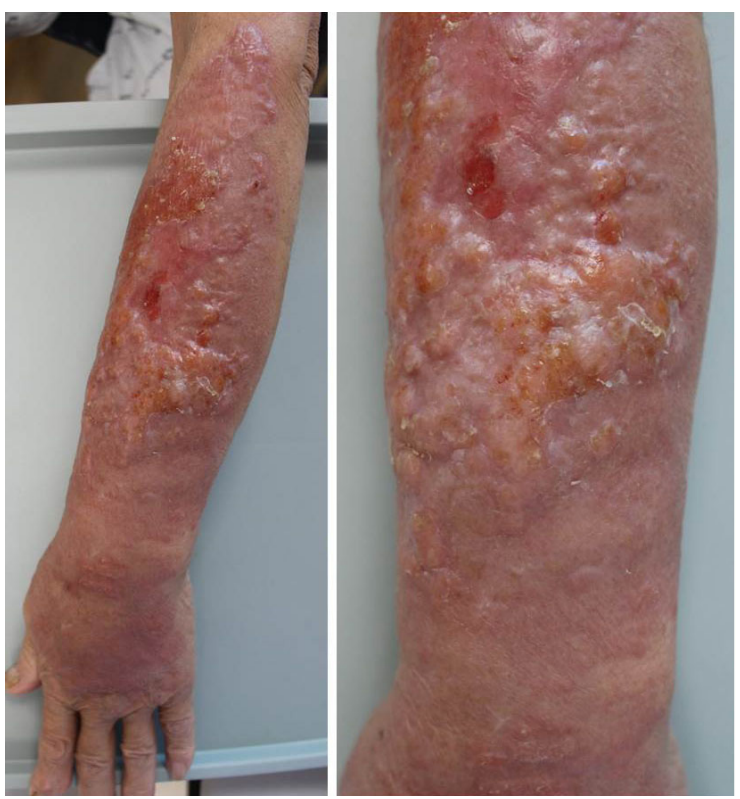

Fig. 1. Erythematous plaque with ulcers and oozing on left forearm. were identified as Cryptococcus neoformans var. grubii by sequence analysis of the internal transcribed spacer gene. Thus, the anti-fungal agent was changed to oral fluconazole $200 \mathrm{mg}$ /day. One week later, however, her symptoms became aggravated, and the dose was increased to $400 \mathrm{mg} /$ day, that maintained for 3 months. After 3 months of follow-up, the lesions improved with post-inflammatory hyperpigmentation (Fig. 3) and without evidence of recurrence.

The informed consent was obtained for the use of patient information and photographs.

\section{DISCUSSION}

PCC is a rare condition that is distinct from secondary cutaneous infections developing after infection of other organs. ${ }^{5)}$ The diagnostic criteria of PCC are confined to the skin and the condition can be diagnosed without clinical evidence of systemic involvement during follow-up for at least 4 weeks after confirmation of $C$. neoformans infection through skin biopsy or culture. ${ }^{1)}$ Neuville et al. $^{2)}$ also reported diagnostic characteristics of PCC, which included rural residency, susceptible environment for trauma, localized lesions, no systemic symptoms, and no antigen detection. Our patient was also living in a rural area, had a history of trauma, and had no systemic symptoms.

The most common skin lesions of PCC are whitlow, cellulitis, and nodules or ulcerations on unclothed areas. ${ }^{2)}$ The lesions in our
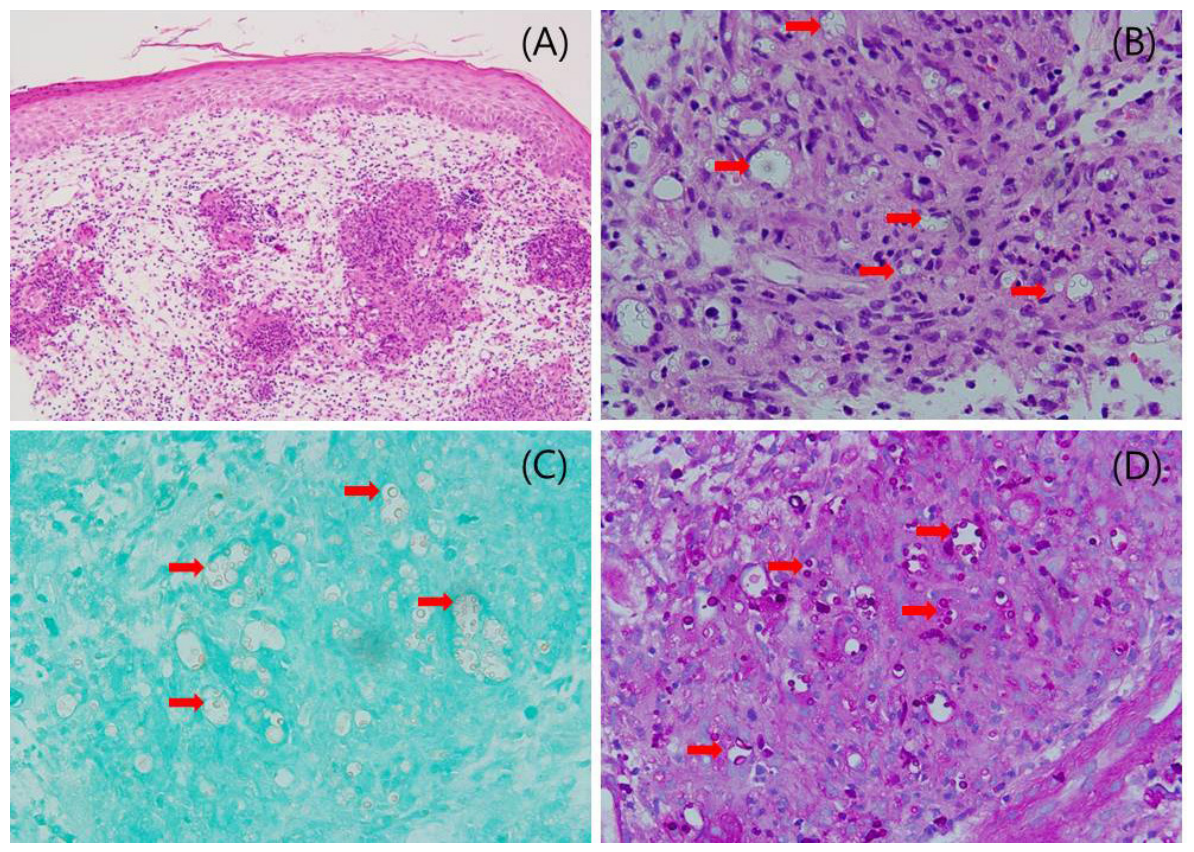

Fig. 2. Histopathologic findings. (A) There was granulomatous inflammation with aggregated histiocytes and multinucleated giant cells in the dermis $(\mathrm{H} \& \mathrm{E}, \times 100)$. (B) Multiple intracytoplasmic yeasts (red arrows) were observed (H\&E, $\times 400)$. (C, D) The yeasts (red arrows) were also identified with Gomori methenamine silver stain (GMS, $\times 400)$ and periodic acid-Schiff stain $(\mathrm{PAS}, \times 400)$. 

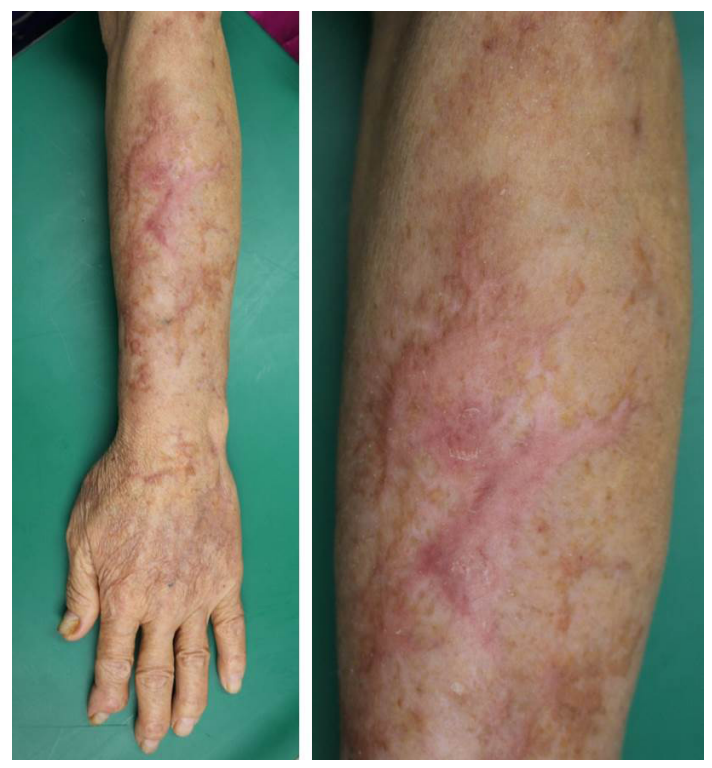

Fig. 3. After 3 months of follow-up. The lesions improved with postinflammatory hyperpigmentation and scar.

patient developed as cellulitis and did not improve with empirical antibiotic treatments. Cutaneous lesions not successfully treated by antibiotics require additional tests to determine whether it is a fungal or mycobacterial infection. In addition, if cryptococcocus is identified by biopsy or culture, other investigations such as chest radiography, thorax CT, serologic tests for HIV, tests of CSF, and blood and urine cultures should be performed to rule out systemic infection, as skin lesions are markers of systemic cryptococcosis. ${ }^{4)}$

PCC is usually treated with fluconazole $(200-400 \mathrm{mg} /$ day $)$, itraconazole (200-400 mg/day), and amphotericin B (0.5-1 mg/ $\mathrm{kg} /$ day), with the dosage and treatment duration depending on the patient's immune status and extent of involvement. ${ }^{3)}$ In the present case, we started treatment with $400 \mathrm{mg}$ of fluconazole and then reduced the dosage to $200 \mathrm{mg}$. We assumed that $200 \mathrm{mg}$ of oral fluconazole would be an adequate therapeutic dose because the bioavailability of oral fluconazole is over $90 \%$ and kidney functions are decreased in older adults. ${ }^{6}$ However, the reduced dose was insufficient considering aggravated symptoms were alleviated by increasing the dosage of fluconazole. We believe that the insufficient dose of fluconazole was due to the necrotic lesions, poor blood supply, insufficient drug exposure at the infection site, and age-related reduction in patient immunity. ${ }^{7,8)}$

Older adults are vulnerable to infections because of reduced $\mathrm{T}$ cell and cytokine activities. ${ }^{8}$ In addition, as the skin microbiome changes with age, the role of the microbiome in inhibiting the growth of fungus may decrease., ${ }^{9,10)}$ The aged population (older than 65 years) in Korea is expected to increase, with corresponding increases in age-related diseases such as infections. ${ }^{9)}$ Therefore, accurate diagnosis and treatment are important and clinicians should be aware that even older adults without underlying diseases are vulnerable to various infectious pathogens.

In this case, because PCC appeared as bacterial cellulitis, it was difficult to clinically suspect this infection. If the lesions do not respond to antibiotics, it is important to perform additional tests to rule out other pathogens, such as fungi. In conclusion, we reported a case of PCC misdiagnosed as cellulitis in an older immunocompetent patient.

\section{ACKNOWLEDGMENTS}

\section{CONFLICT OF INTEREST}

The researchers claim no conflicts of interest.

\section{FUNDING}

This study was supported by grants from the Clinical Medicine Research Institute at Chosun University Hospital in 2019.

\section{AUTHOR CONTRIBUTIONS}

Conceptualization, HC, YIK, BSS; Funding acquisition, HC; Supervision, $\mathrm{HC}$, CHN, MSK, BSS; Writing original draft, HC, YIK; Writing review \& editing, HC, YIK.

\section{REFERENCES}

1. Noble RC, Fajardo LF. Primary cutaneous cryptococcosis: review and morphologic study. Am J Clin Pathol 1972;57:13-22.

2. Neuville S, Dromer F, Morin O, Dupont B, Ronin O, Lortholary $\mathrm{O}$, et al. Primary cutaneous cryptococcosis: a distinct clinical entity. Clin Infect Dis 2003;36:337-47.

3. Saag MS, Graybill RJ, Larsen RA, Pappas PG, Perfect JR, Powderly WG, et al. Practice guidelines for the management of cryptococcal disease. Clin Infect Dis 2000;30:710-8.

4. Kosaraju K, Mukhopadhyay C, Vandana KE, Yagain K, Rao NR. Multiple cutaneous swellings in an immunocompetent host: cryptococcosis overlooked. Braz J Infect Dis 2011;15:394-6.

5. Lu YY, Wu CS, Hong CH. Primary cutaneous cryptococcosis in an immunocompetent man: a case report. Dermatol Sin 2013; 31:90-3.

6. Pfizer Inc. Diflucan (fluconazole) [Internet]. New York, NY: Pfizer Inc.; c2019 [cited 2020 May 16]. Available from: https:// www.pfizer.com/products/product-detail/diflucan.

7. Nucci M, Perfect JR. When primary antifungal therapy fails. Clin Infect Dis 2008;46:1426-33.

8. Choi H, Kim YI, Na CH, Kim MS, Shin BS. Mycobacterium ab- 
scessus skin infection associated with shaving activity in a 75-year-old man. Ann Geriatr Med Res 2018;22:204-7.

9. Choi YJ, Lee DH. Microbiota: a key for healthy aging. Ann Geriatr Med Res 2016;20:168-76.
10. Mayer FL, Kronstad JW. The spectrum of interactions between Cryptococcus neoformans and bacteria. J Fungi (Basel) 2019; $5: 31$. 